\title{
Production and ratios of heavy hadrons from large to small collision systems with a coalescence plus fragmentation approach
}

\author{
Vincenzo Minissale, ${ }^{a, *}$ Salvatore Plumari ${ }^{a, b}$ and Vincenzo Greco ${ }^{a, b}$ \\ a Department of Physics and Astronomy 'E. Majorana', University of Catania, Via S. Sofia 64, \\ 1-95125 Catania, Italy \\ ${ }^{b}$ Laboratori Nazionali del Sud, INFN-LNS, Via S. Sofia 62, I-95123 Catania, Italy \\ E-mail: vincenzo.minissale@lns.infn.it
}

The $\Lambda_{c} / D^{0}$ ratio observed in AA collision at RHIC and LHC energies has a value of the order of the unity. The recent experimental measurements in pp collisions have shown a ratio of $\Lambda_{c} / D^{0} \sim 0.6$, about one order of magnitude larger than that measured in $e^{+} e^{-}, e p$ collisions. We study the hadronization after the propagation of charm quarks in the quarkgluon plasma (QGP). The propagation is described by means of a relativistic Boltzmann transport approach where the non-perturbative interaction between heavy quarks and light quarks is described by means of a quasi-particle approach. Using a coalescence plus fragmentation model for the hadronization we found in heavy-ion collision a large $\Lambda_{c}$ production resulting in a baryon over meson ratio of order $O(1)$. Furthermore we present results for these ratios in pp collisions at top LHC energies assuming the formation of an hot QCD matter at finite temperature. We calculate the heavy baryon/meson ratio and the pT spectra of charmed hadrons with and without strangeness content: $D^{0}, D_{s}$, $\Lambda_{c}, \Sigma_{c}$ and the recently measured $\Xi_{c}$ baryon, finding an enhancement in comparison with the ratio observed for $e^{+} e$, ep collisions; moreover with this approach we predict also a significant production of $\Omega_{c}$ respect to $D^{0}$.

\footnotetext{
*** The European Physical Society Conference on High Energy Physics (EPS-HEP2021), *** *** 26-30 July 2021 ***

*** Online conference, jointly organized by Universität Hamburg and the research center DESY $* * *$
}

${ }^{*}$ Speaker 


\section{Introduction}

The Ultra-relativistic heavy ion collision at Large Hadron Collider (LHC) and at Relativistic Heavy-Ion Collider (RHIC) have provided during the years the possibility to study a new state of matter composed of a strongly interacting plasma of deconfined quark and gluons called Quark-Gluon Plasma (QGP). This state of matter is characterized by the bulk of light-quark and gluons. The heavy quarks can be used as probes of the medium properties because of their large mass. In recent years a lot of studies have done efforts to study the relation between the heavy-quark dynamics in the QGP and the bulk properties [1-10]. In heavy ion collisions the experimental data have shown, in the intermediate region of momenta, a $\Lambda_{c} / D^{0} \sim 0.8 \div 1.5$ at RHIC and $\Lambda_{c} / D^{0} \sim 0.2 \div 0.8$ at LHC. We use a coalescence model that explain the hadronization process through a mechanism that starts from the idea that the final state particles are formed by comoving partons in the QGP that combine their transverse momentum to produce a meson or a baryon with an higher transverse momentum [11-13]. The relative abundance of the different heavy hadron species produced can manifests a baryon-to-meson enhancement for charmed hadrons. [14-17]

Measurements of heavy baryon production in $p p, p A$ and $A A$ collisions from RHIC to top LHC energies have recently attracted more and more attention, currently representing a challenge for the heavy-quark hadronization theoretical understanding. Experimental results from STAR[18] and ALICE [19] collaborations have shown an enhancement of the baryon/meson ratio in the heavy flavor sector like the one observed for light and strange hadrons, larger than the one predicted by the analysis of the charm fragmentation in collision systems as $e^{+} e^{-}, e^{ \pm} p$ and $p p[20]$. On the other hand recently measured ratios in $\mathrm{p}+\mathrm{p}$ collision at LHC at the energy of $\sqrt{s}=5 \mathrm{TeV}$ have shown a $\Lambda_{c} / D^{0}$ ratio larger than the one evaluated in [20]. The production of other single-charmed baryons with content of strangeness, such as $\Xi_{c}$ and $\Omega_{c}$ and their ratios to $D^{0}$ and $\Lambda_{c}$ have been recently presented by ALICE collaboration showing interesting behavior in pp collision [21].

\section{Coalescence plus Fragmentation Model}

The coalescence model used is based on the Wigner formalism. The momentum spectrum of hadrons come from the coalescence integral:

$$
\frac{d^{2} N_{H}}{d P_{T}^{2}}=g_{H} \int \prod_{i=1}^{n} \frac{d^{3} p_{i}}{(2 \pi)^{3} E_{i}} p_{i} \cdot d \sigma_{i} f_{q_{i}}\left(x_{i}, p_{i}\right) f_{H}\left(x_{1} \ldots x_{n}, p_{1} \ldots p_{n}\right) \delta^{(2)}\left(P_{T}-\sum_{i=1}^{n} p_{T, i}\right)
$$

where $d \sigma_{i}$ denotes an element of a space-like hypersurface, $g_{H}$ is the statistical factor to form a colorless hadron while $f_{q_{i}}$ are the quark (anti-quark) phase-space distribution functions for i-th quark (anti-quark). $f_{H}\left(x_{1} \ldots x_{n}, p_{1} \ldots p_{n}\right)$ is the Wigner function and describes the spatial and momentum distribution of quarks within an hadron and can be related to the hadron wave function. In the case of charm hadrons we use a Wigner function that is a Gaussian distribution in relative space and momentum, $f_{M}\left(x_{1}, x_{2} ; p_{1}, p_{2}\right)=A_{W} \exp \left(-\frac{x_{r 1}^{2}}{\sigma_{r}^{2}}-p_{r 1}^{2} \sigma_{r}^{2}\right)$ where $x_{r 1}$ and $p_{r 1}$ are the 4 -vectors for the relative coordinates. The covariant width parameter $\sigma_{r}$ can be related to the oscillator frequency $\omega$ by $\sigma=1 / \sqrt{\mu \omega}$ where $\mu=$ 
$\left(m_{1} m_{2}\right) /\left(m_{1}+m_{2}\right)$ is the reduced mass, and is related to the root mean square charge radius of the meson. For $D^{+}$meson $\left\langle r^{2}\right\rangle_{c h}=0.184 \mathrm{fm}^{2}$ corresponds to a $\sigma_{p}=\sigma_{r}^{-1}=0.283 \mathrm{GeV}$; for $\Lambda_{c}^{+}$the mean square charge radius is $\left\langle r^{2}\right\rangle_{c h}=0.15 \mathrm{fm}^{2}$ with the related widths $\sigma_{p_{1}}=$ $\sigma_{r_{1}}^{-1}=0.251 \mathrm{GeV}$ and $\sigma_{p_{2}}=\sigma_{r_{2}}^{-1}=0.424 \mathrm{GeV}$. We determine the normalization constant $A_{W}$ in order to have, in the limit $p \rightarrow 0$, that all the charm quarks hadronize via coalescence. Once we compute the coalescence probability $P_{\text {coal }}$ for each charm quark, we can assign a probability of fragmentation as $P_{f r a g}\left(p_{T}\right)=1-P_{\text {coal }}\left(p_{T}\right)$. Then we can find that the final hadron fragmentation momentum spectra, from the charm, is given by the convolution of the parton spectrum that do not undergo to coalescence with the fragmentation function $D_{\text {had }}\left(z, Q^{2}\right) \propto 1 /\left[z\left[1-\frac{1}{z}-\frac{\epsilon_{c}}{1-z}\right]^{2}\right]$. In this case we employ the Peterson fragmentation function [22], where $\epsilon_{c}$ is a parameter determined assuring that the experimental data on $D$ and $\Lambda_{c}$ production in $p+p$ collisions, at high momenta, are well described by a fragmentation hadronization mechanism $[4,17]$. The relative fragmentation fractions of charm quarks into different hadron channels are used according to the fractions evaluated in [20].

\subsection{Fireball parameters and quark distribution}

In our model we use a fireball formed by a thermalized system of gluons and $u, d, s$ quarks and anti-quarks at a temperature of $T_{C}=165 \mathrm{MeV}$, that form the bulk of particles. The fireball is considered at $\tau=7.8 \mathrm{fm} / \mathrm{c}$ for $\mathrm{LHC} \mathrm{Pb}+\mathrm{Pb}$ collisions at $\sqrt{s_{N N}}=2.76 \mathrm{TeV}$, and $\tau=4.5 \mathrm{fm} / c$ for RHIC $\mathrm{Au}+\mathrm{Au}$ collisions at $\sqrt{s_{N N}}=200 \mathrm{GeV}$. In $p+p$ collisions at LHC at $\sqrt{s_{N N}}=5.02 \mathrm{TeV}$ we fix the parameter according to hydro-dynamical simulations [23] with $\tau=2.5 \mathrm{fm} / c$. The collective flow is considered assuming a radial flow profile as $\beta_{T}\left(r_{T}\right)=\beta_{\max } \frac{r_{T}}{R}$, where $R$ is the transverse radius of the fireball and $\beta_{\max }$ is the value of the radial flow on the external surface of the fireball. For partons at low transverse momentum, $p_{T}<2 \mathrm{GeV}$, hence we consider a thermal distribution, instead for $p_{T}>2.5 \mathrm{GeV}$, we consider the minijets that have undergone the jet quenching mechanism. For heavy quarks, in heavy
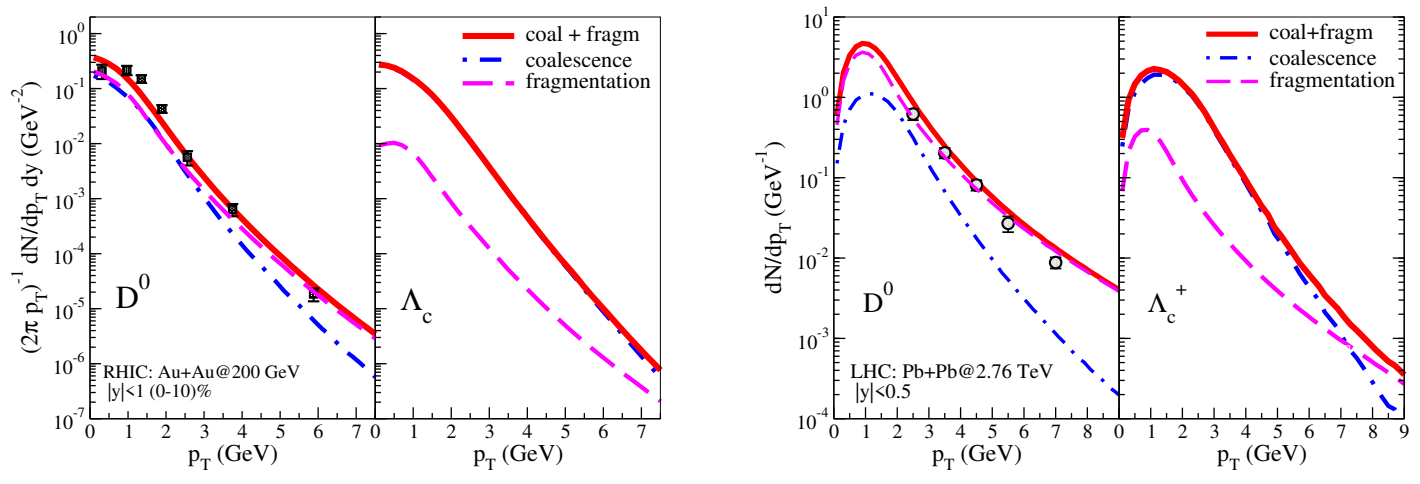

Figure 1: (Color online) (a)(left) Transverse momentum spectra at mid-rapidity for $A u+A u$ collisions at $\sqrt{s}=200 \mathrm{GeV}$ and for $(0-10 \%)$ centrality for $D^{0}$ meson (left panel) and for $\Lambda_{c}^{+}$ baryon (right panel). Experimental data from [25] (b)(right) Transverse momentum spectra at mid-rapidity for $P b+P b$ collisions at $\sqrt{s}=2.76 \mathrm{TeV}$ and for $(0-10 \%)$ centrality for $D^{0}$ meson (left panel) and for $\Lambda_{c}^{+}$baryon (right panel). Experimental data from [26] 
ion collisions, the transverse momentum distribution is obtained by solving the relativistic Boltzmann equation [4] giving a good description of $R_{A A}$ and $v_{2}$ for $D$ mesons. For $p+p$ collisions, the charm quark spectrum have been taken in accordance to Fixed Order + Next-to-Leading Log (FONLL) distribution [24]

\section{Results}

In Fig.1 (a) (left) are shown the transverse momentum spectra at midrapidity for $A u+A u$ collisions at $\sqrt{s}=200 \mathrm{GeV}$ and for $(0-10 \%)$ centrality for $D^{0}$ meson (left panel) and for $\Lambda_{c}^{+}$baryon (right panel). The contribution from coalescence (blue dot-dashed line) and fragmentation (magenta dashed line) for $D^{0}$ production is about similar at momenta in the region at low momenta. The fragmentation contribution becomes dominant for the yield in the region at high momenta. The coalescence (blue dot-dashed line) mechanism appears as the dominant mechanism for the $\Lambda_{c}^{+}$production almost in all the momentum region showed and it is related to the small fragmentation fraction of charm quarks that fragments into this channel, because this fraction is about the $6 \%$ of the total produced heavy hadrons [20]. We have also considered for $\Lambda_{c}^{+}$and $D^{0}$ the yield that comes from the decay of the main hadronic channels, i.e. $D^{* 0}, D^{*+}, \Sigma_{c}^{*}(2520)$ and $\Sigma_{c}(2455)$.

In Fig.1 (b) (right) are shown the transverse momentum spectra at midrapidity for $\mathrm{Pb}+\mathrm{Pb}$ collisions at $\sqrt{s}=2.76 \mathrm{TeV}$ and for $(0-10 \%)$ centrality for $D^{0}$ meson (left panel) and for $\Lambda_{c}^{+}$ baryon (right panel). In this case the contribution from coalescence (blue dot-dashed line) is smaller than the one from fragmentation (magenta dashed line) in all the momentum region considered, thus fragmentation is dominant for the overall yield. For the $\Lambda_{c}^{+}$production the coalescence (blue dot-dashed line) mechanism still appears to be the dominant mechanism up to $p_{T}=7 \mathrm{GeV}$. In the left panel of Fig. 2 we show the $\Lambda_{c}^{+} / D^{0}$ ratio in comparison with the STAR experimental data shown by squares [18]. Our model with coalescence plus

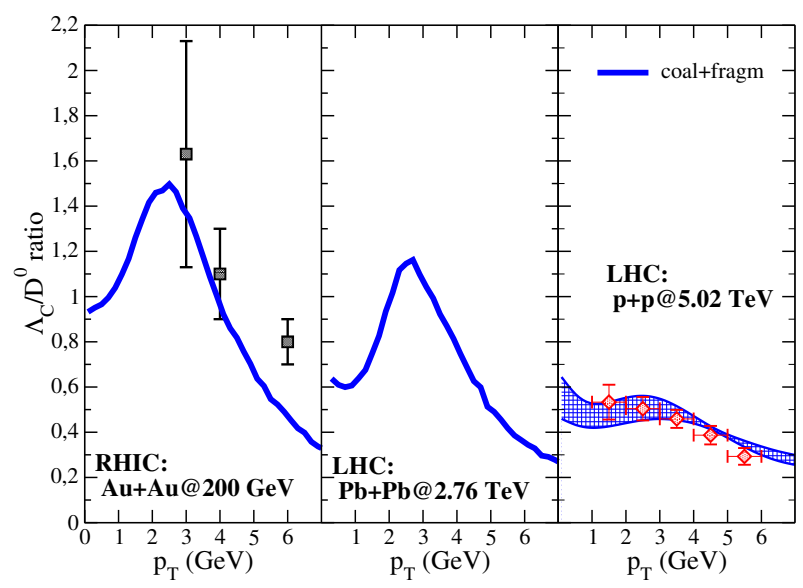

Figure 2: (Color online) $\Lambda_{c}^{+}$to $D^{0}$ ratio as a function of $p_{T}$ and at mid-rapidity for (left panel) $A u+A u$ collisions at $\sqrt{s}=200 \mathrm{GeV}[18]$ for (middle panel) $P b+P b$ collisions at $\sqrt{s}=2.76 \mathrm{TeV}$ and for (right panel) $p p$ collisions at $\sqrt{s}=5 \mathrm{TeV}[28]$ 
fragmentation predicts a rise and fall of the baryon/meson ratio, and we can see a quite good agreement with the experimental data in the peak region. The left and middle panel in Fig.2 show the comparison between RHIC and LHC for the $\Lambda_{c}^{+} / D^{0}$ ratio. The production ratio between coalescence and fragmentation is smaller at LHC than at RHIC as can be seen from the spectra in Fig.1. Therefore, at LHC the larger contribution in particle production from fragmentation leads to a final ratio that is smaller than at RHIC. Recently new experimental data of the $\Lambda_{c}^{+}$to $D^{0}$ ratio have been released for pp collisions at LHC [27, 28], and show an unexpected excess of production of $\Lambda_{c}$ with respect to the simple fragmentation, with values of the ratio of $\sim 0.6$ in the region at low momenta, and a value of $\sim 0.2$ at $10 \mathrm{GeV}$. We have applied our model in the case of $p p$ collisions, assuming the formation of a QGP medium like the one simulated in hydrodynamics calculations [23] and we obtain a good description of the $\Lambda_{c} / D^{0}$ ratio, see Fig.2 (right panel), with the band determined including the uncertainties given by the variation of the Wigner function widths. Our calculations describe the disappearance of the peak, and an enhancement that is significantly different from the ratio obtained with the only fragmentation. In Fig. 3, we show the $\Xi_{c}$ and $\Omega_{c}$ to $D^{0}$ ratios at mid-rapidity in $p p$ collisions at $\sqrt{s}=5.02 \mathrm{TeV}$. In the left panel the $\Omega_{c}^{0} / D^{0}$ ratio is shown. For this ratio we consider $D^{0}$ with both coalescence plus fragmentation contribution, and for the $\Omega_{c}^{0}$ we show three cases, the first considering only the coalescence contribution (green line), the second with both coalescence plus fragmentation (green band) and the third where we include some resonance states in addition to the already considered $\Omega_{c}^{0}(2770)$ (i.e. $\Omega_{c}(3000)^{0}, \Omega_{c}(3005)^{0}, \Omega_{c}(3065)^{0}, \Omega_{c}(3090)^{0}, \Omega_{c}(3120)^{0}$.) (black dash-dotted line). In the right panel, we show the $\Xi_{c} / D^{0}$ ratio (red band) in comparison with recent data from data from ALICE collaboration [21], where we have included the contribution
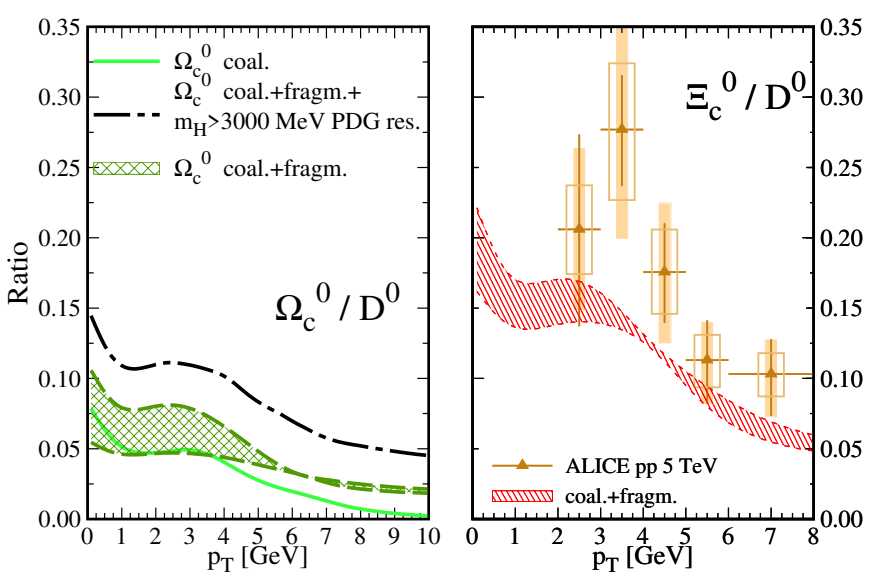

Figure 3: (Color online) (left panel) $\Omega_{c} / D^{0}$ ratio. $\Omega_{c}$ is considered in cases with: only coalescence (green solid line), coalescence plus fragmentation (green band), resonances (black dot-dashed lines). $D^{0}$ contribution comes from both coalescence and fragmentation. (right panel) $\Xi_{c}^{0,+} / D^{0}$ ratios as a function of $p_{T}$ and at mid-rapidity for $p p$ collisions at $\sqrt{s}=5.02 \mathrm{TeV}$ with both coalescence and fragmentation, data from [21]. 
from the principal $\Xi_{c}$ resonances already seen.

\section{References}

[1] He M, Fries R J and Rapp R 2013 Phys. Rev. Lett. 110112301

[2] Uphoff J, Fochler O, Xu Z and Greiner C 2012 Phys. Lett. B717 430-435

[3] Cao S, Qin G Y and Bass S A 2015 Phys. Rev. C92 024907

[4] Scardina F, Das S K, Minissale V, Plumari S and Greco V 2017 Phys. Rev. C96 044905

[5] S. K. Das, M. Ruggieri, F. Scardina, S. Plumari and V. Greco, J. Phys. G 44 (2017) no.9, 095102

[6] Das S K, Plumari S, Chatterjee S, Alam J, Scardina F and Greco V 2017 Phys. Lett. B768 260-264

[7] Das S K, Scardina F, Plumari S and Greco V 2015 Phys. Lett. B747 260-264

[8] Das S K, Torres-Rincon J M, Tolos L, Minissale V, Scardina F and Greco V 2016 Phys. Rev. D94 114039

[9] R. Rapp, et al. Nucl. Phys. A 979 (2018), 21-86

[10] S. Plumari, G. Coci, V. Minissale, S. K. Das, Y. Sun and V. Greco, Phys. Lett. B 805 (2020), 135460

[11] Fries R J, Muller B, Nonaka C and Bass S A 2003 Phys. Rev. Lett. 90202303

[12] Greco V, Ko C and Levai P 2003 Phys.Rev. C68 034904

[13] Minissale V, Scardina F and Greco V 2015 Phys. Rev. C92 054904

[14] Oh Y, Ko C M, Lee S H and Yasui S 2009 Phys. Rev. C79 044905

[15] Plumari S, Minissale V, Das S K, Coci G and Greco V 2018 Eur. Phys. J. C78 348

[16] S. Cho, K. J. Sun, C. M. Ko, S. H. Lee and Y. Oh, Phys. Rev. C 101 (2020) no.2, 024909

[17] V. Minissale, S. Plumari and V. Greco, Phys. Lett. B 821 (2021), 136622

[18] J. Adam et al. [STAR], Phys. Rev. Lett. 124 (2020) no.17, 172301

[19] S. Acharya et al. [ALICE], Phys. Lett. B 793 (2019), 212-223

[20] Lisovyi M, Verbytskyi A and Zenaiev O 2016 Eur. Phys. J. C76 397

[21] S. Acharya et al. [ALICE], [arXiv:2105.05616 [nucl-ex]].

[22] Peterson C, Schlatter D, Schmitt I and Zerwas P M 1983 Phys. Rev. D27 105

[23] R. D. Weller and P. Romatschke, Phys. Lett. B 774 (2017), 351-356

[24] M. Cacciari, S. Frixione, N. Houdeau, M. L. Mangano, P. Nason and G. Ridolfi, JHEP 10 (2012), 137

[25] Adamczyk L et al. (STAR) 2014 Phys. Rev. Lett. 113142301 [Erratum: Phys. Rev. Lett.121,no.22,229901(2018)]

[26] Abelev B et al. (ALICE) 2012 JHEP 09112

[27] A. M. Sirunyan et al. [CMS], Phys. Lett. B 803 (2020), 135328

[28] S. Acharya et al. [ALICE], [arXiv:2011.06079 [nucl-ex]]. 